\title{
Job Rotation as a Learning Mechanism
}

\author{
Jaime Ortega \\ Department of Business Administration, Universidad Carlos III de Madrid, \\ 28903 Getafe, Madrid, Spain \\ jortega@emp.uc3m.es
}

\begin{abstract}
$\mathrm{T}$ his article analyzes the costs and benefits of job rotation as a mechanism with which the firm can learn about the employees' productivities and the profitability of different jobs or activities. I compare job rotation to an assignment policy where employees specialize in one job along their career. The gains from adopting a job rotation policy are larger when there is more prior uncertainty about employees and activities. I argue that this firm learning theory fits the existing evidence on rotation better than alternative explanations based on employee motivation and employee learning.
\end{abstract}

(Job Rotation; Learning)

\section{Introduction}

Recent surveys have revealed that job rotation is used by a significant and increasing number of companies in the United States and other OECD countries. A 1992 U.S. survey by Osterman (1994) reported that $26 \%$ of establishments had more than a half of their core employees involved in job rotation. A later survey by the same author shows that this percentage had increased up to 50\% by 1997 (Osterman 2000). Gittleman et al. (1998) reported that $24 \%$ of establishments with more than 50 employees and $12 \%$ of all establishments in their sample used job rotation. More recently, similar evidence has also become available from other OECD countries (OECD 1999), and the effects of this practice and other human resource innovations on performance are being documented (Ichniowsky et al. 1996, 1997, 1999). Two explanations are usually given to explain job rotation (Campion et al. 1994). Some argue that it is beneficial because it fosters employee learning and increases human capital accumulation. According to this view, rotation gives managers exposure to a variety of experiences and may in this way contribute to their professional development. Others argue that job rotation increases motivation. Rotation is viewed as a mechanism to reduce the employees' boredom and to keep them interested in their job. Both the "employee learning" and the "employee motivation" theories emphasize the effects of job rotation on employees' actions. In this article I argue that the empirical evidence on rotation is not completely consistent with these theories. To better understand the existing empirical evidence, I propose a shift of attention toward the effect of rotation on firm learning, i.e., on the effectiveness with which the employer learns about its employees. I argue that firm learning can better account for the existing empirical evidence than the alternative explanations mentioned above.

Job rotation has important implications for firm learning. On one hand, when employees rotate, the firm receives information about the quality of various job-employee matches, as in Jovanovic (1979). On the other hand, without rotation, the firm receives only direct information about one match, but the information it gets about this one match is very reliable. Thus there is a trade-off between the variety and the number of signals available to the firm for a given employee (Meyer 1994). Moreover, at times the firm may want to learn not only how well different employees match different jobs, but also how profitable different jobs are. This is particularly relevant for companies where innovative production processes are being implemented or new products are being launched. In these cases, we might think that rotation makes it more difficult for the firm to learn 
whether observed productivity differentials between jobs are a result of job-specific or employee-specific components. A policy involving little job rotation, on the other hand, could be the best way to learn about the job-specific component. But it could also be that rotation makes it easier to identify the job-specific components.

In this article I use a statistical learning model to examine these questions. I consider a firm where there is uncertainty about the productivity of different persons at different jobs and about the profitability of different jobs or activities. The firm would like to learn as much as possible in these dimensions to optimally match employees to jobs. Within this framework, I compare two different job assignment policies: A "job rotation" policy, where employees perform a different job in every period, and a "specialization" policy, where each employee is assigned a job at the start and keeps doing this job in subsequent periods. The firm is assumed to choose one of these two allocation policies and commit to it. I study in which cases the firm should adopt one policy or the other.

Empirical evidence on job rotation is scant and must be taken with caution. However, there seem to be two interesting facts. First of all, the empirical evidence suggests that there is a negative correlation between the frequency with which an employee rotates and tenure. Second, the empirical studies of rotation suggest a positive correlation between rotation and the use of new technologies. Using personnel data from the finance department of a large pharmaceutical corporation, Campion et al. (1994) found that the average employee rotated once every two years approximately and that a $1 \%$ increase in tenure produced a $16 \%$ reduction in the frequency of rotation. On the other hand, Gittleman et al. (1998) found that the probability that the average firm in their sample used rotation was $9.6 \%$ if the firm was not using a new technology and increased to $22.3 \%$ if the firm had introduced a new technology during the sample year.

The fact that younger employees rotate more often seems at odds with a motivation theory of job rotation: Newer employees are usually more motivated, and we should therefore expect to observe higher rotation rates when employees have more tenure in the firm. The employee learning theory seems a better explanation: Since newer employees have more to learn, they should rotate more often. However, I argue that the employee learning theory is not necessarily consistent with the positive correlation between innovation and rotation: According to the employee learning theory, an employee should rotate when he has already learned enough about a job; but if a firm has recently adopted a new technology, it should take employees more time to learn about their current assignments. Since employees will not rotate until they have learned their current assignments well, we should expect the rate of rotation to be lower, not higher, when a new technology has recently been adopted. This may contradict the evidence that innovation and job rotation are positively correlated.

The firm learning theory that I propose seems easier to reconcile with the facts. Suppose firms were using rotation to learn more about different employees and activities: Firms could be rotating juniors more frequently than seniors because the abilities of the latter are better known; and rotation could be more profitable when new technologies are introduced because in that case the firm knows less about the profitability of different activities. In this article I use a learning model to study whether this intuition is true: Does the firm learn relatively more with rotation when employees and technologies are newer? I show that this is indeed the case. As the uncertainty regarding employees and activities increases, the relative gains from adopting a job rotation policy increase. First of all, I show that job rotation outperforms specialization as a learning device, i.e., job rotation is more profitable than specialization from a learning point of view. This is consistent with earlier work by Meyer (1994). Second, I show that the relative gains from a job rotation policy are higher when the initial uncertainty about employees is greater (e.g., recently hired employees). Third, I show that these gains are higher when the initial technological uncertainty is higher. Last, I show that the gains are higher when measurement errors are smaller. The first two results can be explained by the fact that the firm always learns more from the first observations of an employee than from subsequent observations. A job rotation policy, where employees move frequently, exploits this feature of learning better than 
a specialization policy. The third result is due to the fact that a job rotation policy protects the firm's learning process from technological uncertainty. If technological uncertainty is not employee-specific, its effect on productivity is differenced out by comparing the performance of two different employees in the same job. The more rotation a firm uses, the better this can be done. Finally, the fourth result is better understood by considering the extreme case where there are no measurement errors. In that case, it suffices to observe an employee once in a certain job to perfectly learn the value of this job-employee match: Additional observations do not add anything. Hence job rotation must be more profitable than specialization. I show that, as measurement errors increase, the gains from rotation become smaller and smaller.

\section{Assumptions and Related Literature}

Job rotation is an interesting mechanism because it makes it possible for the firm to observe employees in action in different positions and thus learn which job fits each employee best. Any meaningful analysis of the problem should therefore take into account that there are significant differences among jobs. This has two different implications. First of all, we should take into account that the employees' human capital is-at least partly-job specific. Thus throughout the paper I assume that the productivity of an employee at a given job depends on his job-specific human capital, which the firm would like to learn. The firm may use job rotation to learn what the best job for each employee is. The second implication is that the surplus generated by different jobs might vary irrespectively of the employees involved. Some activities are intrinsically more productive than others, are better equipped with physical capital, or are more demanded. These differences, however, must also be learned by the firm.

Another important element of the problem is that it is costly to continuously reevaluate job assignments. The information flows that a firm receives about its own employees might be very frequent, but job changes must necessarily be less frequent: Employees cannot be reallocated every time the firm learns something new about them. The problem for the firm, therefore, is to decide whether employees should rotate between two consecutive job evaluations. Firms using job rotation are firms where employees have to move horizontally before they are reevaluated. And firms that do not use job rotation are firms where employees only change jobs when they are reevaluated. Thus in my model I assume that, for some time, the firm has to assign jobs in a predetermined way (either rotating employees or not). Only then can the firm use the information it has learned to modify job assignments.

My article is mostly related to the seminal job matching article of Jovanovic (1979) and to Miller (1984). As in the job matching literature, I consider an environment where the job specificity of human capital plays an important role, and jobs and employees have to be matched in the best way possible. Some employees might be better than others at some jobs, but the productivities of employees at different jobs can only be learned if employees try such jobs: Jobs are experience goods. However, there are also some differences with the job matching literature. Whereas the job matching framework can in principle be applied both to inter- and intrafirm mobility (turnover and rotation, respectively), my focus is on intrafirm mobility: I wish to study whether job rotation policies are an efficient way to learn about one's employees. To pursue this study, I compare a job rotation policy, where employees have to move horizontally before being evaluated, to a specialization policy, where employees do not move until they are evaluated. This approach differs from the job matching literature because in that literature job changes only take place whenever some piece of new, relevant information is learned. In my model, on the contrary, employees change jobs according to a predetermined pattern, and then the firm evaluates the information it has learned to improve job assignments. If employees rotate only when new information is learned, rotation would of course be preferable to no rotation. However, if rotation patterns are predetermined, there is no obvious reason why rotation should be superior. The aim of this article is to analyze this question. 
My basic modeling choices are taken from Meyer (1994). However, the topic of my article is job rotation rather than team composition, and there are, as a consequence, some key differences. First, all human capital in my model is job specific, whereas in Meyer's (1994) all human capital is general. As I have argued, the job-specificity of human capital must be accounted for because firms are interested in rotating employees to see which job fits each of them best. The notion of job-employee fit is meaningful if human capital is job specific. Second, in Meyer (1994) the productivity of employees in different jobs depends on their human capital but does not depend on job characteristics. Instead, I assume that some jobs may be more profitable than others independently of the allocation of employees.

\section{Model and Results}

\subsection{Set-up}

Consider a firm where two persons are employed at two different jobs. To abstract from problems due to team production and keep the symmetry of the problem throughout the analysis, suppose each job has to be performed by only one person at a time, and each person can perform only one job at a time. Thus the firm has to decide who works where at each period. Suppose also that the two employees work for two periods in the firm, and that after that the firm decides to reassign jobs to maximize expected profits. The surplus generated by each activity depends on two factors: The productivity of the employee the job has been assigned to and the profitability of the activity itself. Both are assumed to be constants and are unknown to both the firm and the employees. Let subscripts $i \in\{A, B\}$ and $k \in\{1,2\}$ denote employees and jobs, respectively, and assume that activity $k^{\prime} s$ production function in period $t \in\{1,2\}$ is

$$
y_{k t}=\eta_{i k}+\theta_{k}+\varepsilon_{k t},
$$

where $\eta_{i k}+\theta_{k}$ is the expected productivity of employee $i$ in job $k$ and $\varepsilon_{k t}$ is a productivity shock. The term $\eta_{i k}$ denotes the part of employee $i$ 's human capital that is specific to job $k$. All job-specific human capital is assumed to be firm specific as well. I assume that there is a competitive labor market that learns about the employees' general human capital at the same speed as the firm itself. As a consequence, there is no need to introduce a general human capital term in Equation (1), and the value of the firm is assumed to depend only on the levels of specific human and nonhuman capital, as well as an exogenous shock. The level of nonhuman capital that is needed in job $k$ is represented by $\theta_{k}$, and the a priori beliefs about all productivity components are assumed to be normally distributed: Specifically, I assume that $\eta_{i k} \sim$ $N\left(0, \sigma_{\eta}^{2}\right), \theta_{k} \sim N\left(0, \sigma_{\theta}^{2}\right)$, and $\varepsilon_{k t} \sim N\left(0, \sigma_{\varepsilon}^{2}\right)$. For simplicity I assume that all these variables are a priori independently distributed. This assumption is relaxed in $\S 3.3$.

\subsection{Job Assignment Policies}

Ideally, an optimal job assignment policy would require the firm to reallocate jobs whenever new information alters the existing ranking of jobemployee matches. Jobs would have to be reallocated as frequently as such new information would be learned. There are, however, limits to the frequency with which a company can afford to undertake such reallocations. One limit is that it is costly to move employees around: Employees have to adapt to new responsibilities, learn to work with new people, or spend time training their successors. Second, the process of evaluating new information to assess which reallocations are more profitable is also costly. Last, an allocation policy that is very sensitive to new information might create an incentive for employees to exert influence activities (Milgrom 1988) and to try to manipulate such information. Let $\Pi_{R}$ and $\Pi_{S}$ be the values of firms following a job rotation and a specialization policy, respectively. The gain from adopting a job rotation policy is $\Delta=\Pi_{R}-\Pi_{S}$. We should expect a job rotation policy to be adopted when $\Delta>C$, where $C$ are the above-mentioned costs of implementing job rotation. To focus on the learning aspects of both policies, I assume that $C$ is exogenous and try to characterize the firms for which it is more likely to have $\Delta>C$. We begin by computing $\Pi_{S}$ and $\Pi_{R}$.

3.2.1. Specialization. I consider a specialization policy first: Each employee performs the same job for 
two consecutive periods. I use this as a benchmark to compare a job rotation policy to. Without loss of generality I assume that employee $A$ is assigned Job 1 and employee $B$ is assigned Job 2, and therefore

$$
\begin{aligned}
& y_{1 t}=\eta_{A 1}+\theta_{1}+\varepsilon_{1 t}, \\
& y_{2 t}=\eta_{B 2}+\theta_{2}+\varepsilon_{2 t}
\end{aligned}
$$

for $t=1,2$. The firm's expected value in this case is

$$
\begin{gathered}
\Pi_{S}=E_{0}\left[\operatorname { m a x } \left\{E_{2}\left(\eta_{A 1}+\theta_{1}\right)+E_{2}\left(\eta_{B 2}+\theta_{2}\right),\right.\right. \\
\left.\left.E_{2}\left(\eta_{B 1}+\theta_{1}\right)+E_{2}\left(\eta_{A 2}+\theta_{2}\right)\right\}\right],
\end{gathered}
$$

where $E_{t}(\cdot)$ is the expectation operator conditional on all information available at the end of period $t$. In principle, we would have to add to this expression the value of the surplus generated in Periods 1 and 2, while the firm is learning. However, the expected value of this surplus will be the same for the specialization and the rotation policies, because all employees and jobs have been assumed to be ex ante identical. Note also that, because of specialization, there is one dimension of each employee's human capital that the firm learns nothing about. Hence $E_{2}\left(\eta_{A 2}\right)=E_{0}\left(\eta_{A 2}\right)=0$ and $E_{2}\left(\eta_{B 1}\right)=E_{0}\left(\eta_{B 1}\right)=0$. As a consequence this expression simplifies to

$$
\Pi_{S}=E_{0}\left[\max \left\{E_{2}\left(\eta_{A 1}\right)+E_{2}\left(\eta_{B 2}\right), 0\right\}\right] .
$$

By specializing, the firm chooses to learn a lot about a few dimensions instead of learning a little about more dimensions. Expression (4) is simply the expected value of a truncated normal variable and the following result can be easily proven:

Lemma 1. The expected value of the specialization policy is

$$
\Pi_{S}=\sqrt{\frac{2}{\pi}}\left(\frac{\sigma_{\eta}^{4}}{\sigma_{\varepsilon}^{2}+2 \sigma_{\eta}^{2}+2 \sigma_{\theta}^{2}}\right)^{1 / 2} .
$$

Proof. See Appendix.

The expected value of a specialization policy is decreasing in $\sigma_{\varepsilon}^{2}$. Note that the value of each assignment policy depends on how much the firm learns about its employees. When $\sigma_{\varepsilon}^{2}$ is high, there is little learning and, as a consequence, the value of the firm is lower. On the other hand, the value of specialization is increasing in $\sigma_{\eta}^{2}$ because, when $\sigma_{\eta}^{2}$ is higher, there is more information to be learned on the employee dimension, and the value of any policy that allows learning in that dimension should be higher. We will later see that the value of job rotation is also decreasing in $\sigma_{\varepsilon}^{2}$ and increasing in $\sigma_{\eta}^{2}$. Finally, specialization has a lower value when there is more prior uncertainty regarding the profitability of different jobs. This is so because the uncertainty regarding the job-specific component of productivity (the $\theta$ s) simply adds more noise to the inference of individual abilities.

3.2.2. Job Rotation. Suppose now that the firm follows a job rotation policy: Each employee works at a different job in each of the two periods. Without loss of generality, suppose $A$ starts doing Job 1 and is then moved to Job 2, whereas $B$ starts at Job 2 and is then moved to Job 1 . The production technology is then

$$
\begin{aligned}
& y_{11}=\eta_{A 1}+\theta_{1}+\varepsilon_{11}, \\
& y_{21}=\eta_{B 2}+\theta_{2}+\varepsilon_{21}, \\
& y_{12}=\eta_{B 1}+\theta_{1}+\varepsilon_{12}, \\
& y_{22}=\eta_{A 2}+\theta_{2}+\varepsilon_{22} .
\end{aligned}
$$

At the end of Period 2, each employee has worked in each job once, jobs are optimally reassigned, and the ex-ante value of the firm is

$$
\begin{gathered}
\Pi_{R}=E_{0}\left[\operatorname { m a x } \left\{E_{2}\left(\eta_{A 1}+\theta_{1}\right)+E_{2}\left(\eta_{B 2}+\theta_{2}\right),\right.\right. \\
\left.\left.E_{2}\left(\eta_{B 1}+\theta_{1}\right)+E_{2}\left(\eta_{A 2}+\theta_{2}\right)\right\}\right] .
\end{gathered}
$$

This is the expected value of the maximum of two correlated normal variables. The following lemma is proved in the appendix:

Lemma 2. The expected value of the job rotation policy is

$$
\Pi_{R}=\sqrt{\frac{2}{\pi}}\left(\frac{\sigma_{\eta}^{4}}{\sigma_{\varepsilon}^{2}+\sigma_{\eta}^{2}}\right)^{1 / 2} .
$$

Proof. See Appendix.

As in the specialization case, the value of the firm is decreasing in $\sigma_{\varepsilon}^{2}$ and increasing in $\sigma_{\eta}^{2}$. The intuition 
is the same as in the specialization case: The value of the firm under a job rotation policy is lower when abilities are more difficult to measure and is higher when the initial information about the employees is less precise (and hence there is more to be learned by having them rotate). On the other hand, the value of job rotation does not depend on the job-specific component of productivity $\left(\sigma_{\theta}^{2}\right)$. This is so because, with rotation, the firm can eliminate the effect of technological, job-specific uncertainty on productivity by simply comparing the performance of two different employees at the same job. In other words, with job rotation the firm can better use relative performance evaluation.

3.2.3. Comparison. Now consider the profit differential between the two human resource practices, $\Delta=\Pi_{R}-\Pi_{S}$.

Proposition 1. For every value of $\sigma_{\varepsilon}^{2}, \sigma_{\eta}^{2}$ and $\sigma_{\theta}^{2}$ the profit differential $\Delta$ is strictly positive, increasing in $\sigma_{\eta}^{2}$ and $\sigma_{\theta}^{2}$, and decreasing in $\sigma_{\varepsilon}^{2}$.

Proof. See Appendix.

The result that $\Delta>0$ means that, from a learning point of view only, job rotation outperforms specialization. This result is consistent with Meyer's (1994) argument that, in her model, the introduction of job rotation would improve learning. This result is because the firm always learns more from the first observation of a particular job-employee match than from subsequent observations. With a job rotation policy, every time the firm learns something, it learns it for the first time. On the other hand, with a specialization policy the firm learns twice for the first time (in Period 1) and twice for the second time (in Period 2). The result with respect to $\sigma_{\theta}^{2}$ is straightforward: With a job rotation policy, the uncertainty about the job component of productivity has no effect on learning. This effect can be differenced out by undertaking relative performance evaluation. However, $\sigma_{\theta}^{2}$ has a negative effect on learning if the firm is following a specialization policy for reasons already explained.

The other two results are less evident: Both $\Pi_{R}$ and $\Pi_{S}$ are decreasing in $\sigma_{\varepsilon}^{2}$ and increasing in $\sigma_{\eta}^{2}$. To get some intuition about this, first look at the case where $\sigma_{\varepsilon}^{2}$ would be zero, i.e., a hypothetical situation where there would be no measurement errors. In this kind of situation, after Period 1 ended the firm would have already learned all it could have learned about the initial job allocation. Since there would be nothing more to learn, it would be better to have employees rotate in the second period. If we now took a slightly higher value of $\sigma_{\varepsilon}^{2}$, job rotation would still be more profitable than specialization, but it would be a little bit less profitable than before. The proof shows that this intuition extends to all values of $\sigma_{\varepsilon}^{2}$ : The profit differential is decreasing in $\sigma_{\varepsilon}^{2}$. Finally, consider the comparative statics with respect to $\sigma_{\eta}^{2}$. The values of both allocation policies are increasing in $\sigma_{\eta}^{2}$ because both policies allow the firm to learn more, and the value of learning is higher when the prior uncertainty is higher. However, the value of job rotation is more sensitive to the initial level of uncertainty because, with this policy, every piece of information that the firm learns is completely new: Every observation that the firm has access to refers to a dimension for which the firm did not have any information other than its prior beliefs.

\subsection{Correlated Abilities}

In reality, the productivities of a same employee in different jobs are likely to be positively correlated: An employee who performs well in one of the jobs is likely to perform well in other jobs too. To take this into account I now assume that the covariance between $\eta_{i 1}$ and $\eta_{i 2}$ is equal to $\tau>0$ for every $i \in$ $\{A, B\}$. I keep all other assumptions unchanged. We can follow the same steps as before and find that the expected value of a specialization policy is

$$
\Pi_{S}=\sqrt{\frac{2}{\pi}} \frac{\sigma_{\eta}^{2}-\tau}{\sqrt{\sigma_{\varepsilon}^{2}+2\left(\sigma_{\eta}^{2}+\sigma_{\theta}^{2}\right)}} .
$$

Details are given in the Appendix. As in Lemma 1, the value of specialization is decreasing in $\sigma_{\varepsilon}^{2}$ and $\sigma_{\theta}^{2}$ and increasing in $\sigma_{\eta}^{2}$. The reasons for these results are similar to the ones for the case where $\tau=0$. Besides, we can now see the effect on $\Delta$ of the positive correlation of abilities. An increase in this correlation reduces the gains from a specialization policy. The reason for this is that, when there is a higher positive correlation, there is less to be learned with either policy (as shown below, the value of rotation is also decreasing in $\tau$ ). 
The expected value of the job rotation policy is

$$
\Pi_{R}=\sqrt{\frac{2}{\pi}} \frac{\sigma_{\eta}^{2}-\tau}{\sqrt{\sigma_{\varepsilon}^{2}+\sigma_{\eta}^{2}-\tau}} .
$$

This also shows the robustness of our previous findings: The value of specialization does not depend on $\sigma_{\theta}^{2}$ and is decreasing in $\sigma_{\varepsilon}^{2}$, increasing in $\sigma_{\eta}^{2}$ and decreasing in $\tau$. The reasons are similar to the ones discussed above.

As shown in the appendix, comparative statics of $\Delta$ are also robust to the positive correlation of abilities. I prove that $\Delta$ is strictly positive, increasing in $\sigma_{\eta}^{2}$ and $\sigma_{\theta}^{2}$, and decreasing in $\sigma_{\varepsilon}^{2}$. This means that all the qualitative predictions of the model with uncorrelated abilities remain unchanged in the correlated case.

\section{Discussion and Conclusions}

At present, empirical evidence on job rotation needs to be taken with caution because it is not complete. However, the predictions we have derived are consistent with the evidence we have at this point. Using a 1993 sample of over 5,800 U.S. establishments from all industries, Gittleman et al. (1998) found that firms using new technologies were significantly more likely to use job rotation. Unpublished results obtained for their paper reveal that an average establishment in their sample had a $9.6 \%$ probability of using job rotation, whereas an average establishment that had introduced a new technology during the sample year had a $22.3 \%$ probability. My model shows that job rotation is in fact a better learning mechanism than specialization when there is little prior information about the relative profitability of different activities. The prediction that rotation is more profitable than specialization when employees are in their early careers is also consistent with the empirical evidence, although in this case the empirical evidence is less representative. Campion et al. (1994) used personnel data from the finance department of a single large corporation that was using rotation extensively. They found that the frequency of rotation drastically diminished with tenure.

As I mentioned before, two other explanations have been given for the use of job rotation. According to the employee motivation theory, firms make employees rotate to reduce their boredom and make them more interested in their work. Despite its intuitive appeal, this theory seems at odds with the available empirical evidence. It seems that more innovative firms use more job rotation. However, in a company where more innovative practices are implemented the nature of jobs is newer and employees are less likely to be bored. In these cases employees are required to understand and solve new problems even if they stay at their jobs. Moreover, it is usually thought that motivational problems are more likely to arise rather late in the employees' careers. As a consequence, if rotation was mostly playing a motivational role we would expect employees with more tenure to rotate more than those with less tenure. However, the empirical evidence suggests that the correlation between tenure and rotation is significantly negative, not positive.

In view of the existing empirical evidence, the employee learning theory seems more plausible. According to that theory firms make employees rotate so that they acquire new skills. If employees with less tenure have comparatively less human capital, they should rotate more. This would explain the negative correlation between tenure and rotation. Consider, however, the predictions of this theory regarding innovation. First of all, if a company undertakes continuous innovation, it is not clear that low-tenure employees will have more need to learn than hightenure employees: On one hand, a large proportion of the human capital that high-tenure employees accumulated might have lost its value; on the other hand, newly hired employees might have learned many of the new skills that are required in the firm at school. Hence, it is not clear that in innovative firms tenure and rotation should be negatively correlated. Finally, consider a comparison between innovative and noninnovative companies. It is beyond doubt that an innovative company will have a greater need to induce its employees to learn. However, why should rotation be the right way to induce such learning? If substantial innovation is being undertaken, then each employee will learn new skills even if he does not rotate. The idea behind the employee learning theory is that employees should change jobs when they have already learned enough about their old job. However, if a company has just introduced a new technology, employees might need more time to learn all 


\section{ORTEGA}

Job Rotation as a Learning Mechanism

they need to learn about their current job, and it will take them more time to be ready to rotate. We should then expect innovation and rotation to be negatively correlated.

The explanation based on firm learning seems to be able to accommodate the two pieces of empirical evidence at the same time: As we have seen, the advantage of a rotation policy over a specialization policy increases as prior uncertainty regarding employees and activities increases. This means that it is more profitable for the firm to rotate employees with low rather than high tenure and that rotation is more profitable in more innovative activities. When firms are very innovative, employees would learn a lot even if they did not change jobs. However, these firms prefer to have them rotate because in this way the firms can better learn how well suited different persons are to different activities. On the other hand, firm learning would also explain why high-tenure employees rotate less even though they are more likely to be less motivated: The firm simply does not need to learn much about them.

\section{Acknowledgments}

The auhor is grateful to Maury Gittleman for providing unpublished results obtained for his paper with Michael Horrigan and Mary Joyce (Gittleman et al. 1998). He would also like to thank Susan Athey, Bengt Holmstrom, seminar participants at CLS (University of Aarhus and Aarhus Business School), IZA (Bonn), and SITE (Stockholm School of Economics), Departmental Editor Linda Argote, an anonymous associate editor, and two anonymous referees.

\section{Appendix}

\section{General Updating Formula}

Subsequent results in this appendix make use of the following statistical result:

Lemma 3. Let $y_{1}=L \eta+\varepsilon_{1}$, where $\varepsilon_{1} \sim N\left(0, G_{1}^{-1}\right)$ and $\eta \sim$ $N\left(\eta_{0}, H_{0}^{-1}\right) ; y_{1}, \eta$, and $\varepsilon_{1}$ are $n \times 1, m \times 1$, and $m \times 1$ vectors (respectively); $L$ is an $m \times n$ matrix; and $G_{1}$ and $H_{0}$ are symmetric $m \times m$ and $n \times n$ (respectively) matrices. Then the posterior distribution of $\eta$ conditional on $y_{1}$ is $N\left(\eta_{1}, H_{1}^{-1}\right)$, where

$$
\begin{aligned}
& \eta_{1}=\left(H_{0}+L^{\prime} G_{1} L\right)^{-1}\left(H_{0} \eta_{0}+L^{\prime} G_{1} y_{1}\right), \\
& H_{1}=H_{0}+L^{\prime} G_{1} L .
\end{aligned}
$$

Proof. The proof is standard and is ommitted for the sake of concision. This result is a generalization of the theorem in $\$ 16.4 .1$ (pp. 382-383) of Pratt et al. (1995).

Proof of Lemma 1. First, note that $\Pi_{S}$ (Expression (4) in §2.2) is proportional to the expected value of a truncated normal variable. The coefficient of proportionality is $1 / 2$ :

$$
\begin{aligned}
\Pi_{S} & =\frac{1}{2}\left(E_{0}\left[E_{2}\left(\eta_{A 1}+\eta_{B 2}\right)\right]+2 \varphi(0) \sqrt{V_{0}\left[E_{2}\left(\eta_{A 1}+\eta_{B 2}\right)\right]}\right) \\
& =(2 \pi)^{-1 / 2} \sqrt{V_{0}\left[E_{2}\left(\eta_{A 1}+\eta_{B 2}\right)\right]},
\end{aligned}
$$

where $\varphi(\cdot)$ is the density function of a standard univariate normal variable and $V_{t}(\cdot)$ is the variance operator conditional on all information available at the end of period $t$.

Second, for any normal random variable $x$ with $E_{0}(x)=0$ we can prove that $V_{0}\left[E_{2}(x)\right]=V_{0}(x)-V_{2}(x)$. Letting $I_{t}$ denote all information available at the end of period $t$, and using (a) the law of iterated expectations and (b) the fact that, ex ante, the variance of a posterior normal variable is not a random variable, we have

$$
\begin{aligned}
V_{0}\left[E_{2}(x)\right] & =V\left[E\left(x \mid I_{2}\right) \mid I_{0}\right]=E\left[E\left(x \mid I_{2}\right)^{2} \mid I_{0}\right]-E\left[E\left(x \mid I_{2}\right) \mid I_{0}\right]^{2} \\
& =E\left[E\left(x \mid I_{2}\right)^{2} \mid I_{0}\right]-E\left(x \mid I_{0}\right)^{2}=E\left[E\left(x \mid I_{2}\right)^{2} \mid I_{0}\right] \\
& =E\left[E\left(x^{2} \mid I_{2}\right)-V\left(x \mid I_{2}\right) \mid I_{0}\right] \\
& =E\left[E\left(x^{2} \mid I_{2}\right) \mid I_{0}\right]-E\left[V\left(x \mid I_{2}\right) \mid I_{0}\right] \\
& =E\left(x^{2} \mid I_{0}\right)-V\left(x \mid I_{2}\right)=V\left(x \mid I_{0}\right)-V\left(x \mid I_{2}\right) \\
& =V_{0}(x)-V_{2}(x) .
\end{aligned}
$$

Third, by assumption we have $V_{0}\left(\eta_{A 1}+\eta_{B 2}\right)=2 \sigma_{\eta}^{2}$.

Fourth, we can use Lemma 3 to compute the posterior variance in the specialization case:

$$
V_{2}\left(\eta_{A 1}+\eta_{B 2}\right)=\frac{2 \sigma_{\eta}^{2} \sigma_{\theta}^{2} \sigma_{\varepsilon}^{2}\left(\sigma_{\eta}^{2} \sigma_{\varepsilon}^{2}+2 \sigma_{\eta}^{2} \sigma_{\theta}^{2}\right)}{\left(\sigma_{\eta}^{2} \sigma_{\varepsilon}^{2}+2 \sigma_{\eta}^{2} \sigma_{\theta}^{2}\right)\left(\sigma_{\theta}^{2} \sigma_{\varepsilon}^{2}+2 \sigma_{\eta}^{2} \sigma_{\theta}^{2}\right)-4 \sigma_{\eta}^{4} \sigma_{\theta}^{4}} .
$$

Finally, combining these results gives Lemma 1.

Proof of Lemma 2. To find the value of job rotation we use Afonja (1972), which provides an explicit solution: If $x=\left(x_{1}, x_{2}\right)$ is a bivariate normal variable with $E\left(x_{1}\right)=E\left(x_{2}\right)=m$ and $V(x)=\left\{\sigma_{i j}\right\}$ (for $i, j \in\{1,2\}$ ), then the expectation of the maximum is

$$
E[\max (x)]=m+(2 \pi)^{-1 / 2} \sqrt{\sigma_{11}+\sigma_{22}-2 \sigma_{12}} .
$$

Now defining $y_{A B}=E_{2}\left(\eta_{A 1}+\theta_{1}+\eta_{B 2}+\theta_{2}\right)$ and $y_{B A}=E_{2}\left(\eta_{B 1}+\theta_{1}+\right.$ $\left.\eta_{A 2}+\theta_{2}\right)$, we have

$$
\Pi_{R}=(2 \pi)^{-1 / 2} \sqrt{V_{0}\left(y_{A B}-y_{B A}\right)} .
$$

By symmetry, $V_{0}\left(y_{A B}\right)=V_{0}\left(y_{B A}\right)$. Furthermore, applying (13), we have $V_{0}\left(y_{A B}\right)=V_{0}\left(\eta_{A 1}+\theta_{1}+\eta_{B 2}+\theta_{2}\right)-V_{2}\left(\eta_{A 1}+\theta_{1}+\eta_{B 2}+\theta_{2}\right)$ and $\operatorname{cov}_{0}\left(y_{A B}, y_{B A}\right)=\operatorname{cov}_{0}\left(\eta_{A 1}+\theta_{1}+\eta_{B 2}+\theta_{2}, \eta_{B 1}+\theta_{1}+\eta_{A 2}+\theta_{2},\right)-$ $\operatorname{cov}_{2}\left(\eta_{A 1}+\theta_{1}+\eta_{B 2}+\theta_{2}, \eta_{B 1}+\theta_{1}+\eta_{A 2}+\theta_{2}\right)$, where $\operatorname{cov}_{t}(.,$.$) is the$ covariance operator conditional on all information available at the end of period $t$. 
Given our distributional assumptions, we have $V_{0}\left(\eta_{A 1}+\theta_{1}+\right.$ $\left.\eta_{B 2}+\theta_{2}\right)=2 \sigma_{\eta}^{2}+2 \sigma_{\theta}^{2}$. Moreover, Bayesian updating gives the following expressions for the posterior variance and covariance:

$$
\begin{gathered}
V_{2}\left(\eta_{A 1}+\theta_{1}+\eta_{B 2}+\theta_{2}\right)=\frac{2 \sigma_{\varepsilon}^{2}\left[\sigma_{\varepsilon}^{2}\left(\sigma_{\eta}^{2}+\sigma_{\theta}^{2}\right)+\sigma_{\eta}^{2}\left(\sigma_{\eta}^{2}+2 \sigma_{\theta}^{2}\right)\right]}{\left(\sigma_{\eta}^{2}+\sigma_{\varepsilon}^{2}\right)\left(\sigma_{\eta}^{2}+\sigma_{\varepsilon}^{2}+2 \sigma_{\theta}^{2}\right)}, \\
\operatorname{cov}_{2}\left(\eta_{A 1}+\theta_{1}+\eta_{B 2}+\theta_{2}, \eta_{B 1}+\theta_{1}+\eta_{A 2}+\theta_{2}\right) \\
=\frac{2 \sigma_{\theta}^{2} \sigma_{\varepsilon}^{4}}{\left(\sigma_{\eta}^{2}+\sigma_{\varepsilon}^{2}\right)\left(\sigma_{\eta}^{2}+\sigma_{\varepsilon}^{2}+2 \sigma_{\theta}^{2}\right)} .
\end{gathered}
$$

Combining these results, we get

$$
V_{0}\left(y_{A B}-y_{B A}\right)=\frac{\sigma_{\eta}^{4}}{\sigma_{\eta}^{2}+\sigma_{\varepsilon}^{2}}
$$

and Lemma 2 follows.

Proof of Proposition 1. By Lemmas 1 and 2, the difference in profits between a job rotation and a specialiation policies is

$$
\Delta=\sqrt{\frac{2}{\pi}}\left[\left(\frac{\sigma_{\eta}^{4}}{\sigma_{\varepsilon}^{2}+\sigma_{\eta}^{2}}\right)^{1 / 2}-\left(\frac{\sigma_{\eta}^{4}}{\sigma_{\varepsilon}^{2}+2 \sigma_{\eta}^{2}+2 \sigma_{\theta}^{2}}\right)^{1 / 2}\right] .
$$

The proof that $\Delta>0$ is straightforward. To prove the rest of the proposition, it suffices to differentiate with respect to $\sigma_{\varepsilon}^{2}, \sigma_{\eta}^{2}$, and $\sigma_{\theta}^{2}$. Differentiating with respect to $\sigma_{\varepsilon}^{2}$, we find

$$
\frac{\partial \Delta}{\partial \sigma_{\varepsilon}^{2}}=\sqrt{\frac{1}{2 \pi}} \sigma_{\eta}^{2}\left[\left(\sigma_{\varepsilon}^{2}+2 \sigma_{\eta}^{2}+2 \sigma_{\theta}^{2}\right)^{-3 / 2}-\left(\sigma_{\eta}^{2}+\sigma_{\varepsilon}^{2}\right)^{-3 / 2}\right]<0 .
$$

Differentiation with respect to $\sigma_{\eta}^{2}$ yields

$$
\frac{\partial \Pi_{R}}{\partial \sigma_{\eta}^{2}}=\sqrt{\frac{1}{2 \pi}} \frac{\sigma_{\eta}^{2}+2 \sigma_{\varepsilon}^{2}}{\left(\sigma_{\eta}^{2}+\sigma_{\varepsilon}^{2}\right)^{3 / 2}}
$$

and

$$
\frac{\partial \Pi_{S}}{\partial \sigma_{\eta}^{2}}=\sqrt{\frac{2}{\pi}} \frac{\sigma_{\varepsilon}^{2}+\sigma_{\eta}^{2}+2 \sigma_{\theta}^{2}}{\left(\sigma_{\varepsilon}^{2}+2 \sigma_{\eta}^{2}+2 \sigma_{\theta}^{2}\right)^{3 / 2}} .
$$

Letting $\alpha=\sigma_{\eta}^{2} / \sigma_{\varepsilon}^{2}$ and $\beta=\sigma_{\theta}^{2} / \sigma_{\varepsilon}^{2}$, we have

$$
\begin{aligned}
\frac{\partial \Pi_{R}}{\partial \sigma_{\eta}^{2}}> & \frac{\partial \Pi_{S}}{\partial \sigma_{\eta}^{2}} \Leftrightarrow\left(\frac{\alpha+2}{\alpha+2 \beta+1}\right)^{2}\left(\frac{2 \alpha+2 \beta+1}{\alpha+1}\right)^{3}>4 \\
\Leftrightarrow & (\alpha+2)^{2}(2 \alpha+2 \beta+1)^{3}-4(\alpha+2 \beta+1)^{2}(\alpha+1)^{3}>0 \\
\Leftrightarrow & 4 \alpha^{5}+8 \alpha^{4}(\beta+3)+8 \beta(2 \beta+1)^{2}+2 \alpha^{3}\left(4 \beta^{2}+28 \beta+23\right) \\
& +8 \alpha\left(4 \beta^{3}+12 \beta^{2}+7 \beta+1\right) \\
& +\alpha^{2}\left(8 \beta^{3}+6 \beta^{2}+102 \beta+33\right)>0,
\end{aligned}
$$

which is satisfied. Hence $\Delta$ is increasing in $\sigma_{\eta}^{2}$.

Finally, differentiation with respect to $\sigma_{\theta}^{2}$ gives us the following result:

$$
\frac{\partial \Delta}{\partial \sigma_{\theta}^{2}}=-\frac{\partial \Pi_{S}}{\partial \sigma_{\theta}^{2}}=\sqrt{\frac{2}{\pi}} \frac{\sigma_{\eta}^{2}}{\left(\sigma_{\varepsilon}^{2}+2 \sigma_{\eta}^{2}+2 \sigma_{\theta}^{2}\right)^{3 / 2}}>0 .
$$

\section{Proofs for the Correlated Case}

The procedure to find the value of each job allocation policy is similar to the one followed for the uncorrelated case. We use Lemma 3 to compute the posterior variances and then use Afonja's (1972) result (Equation (15)) to compute the expected value of each allocation policy.

The proof that $\Delta>0$ is straightforward. The comparative statics are obtained by differentiation. First of all, $\Delta$ is clearly increasing in $\sigma_{\theta}^{2}: \Pi_{R}$ does not depend on $\sigma_{\theta}^{2}$ and $\Pi_{S}$ is decreasing in $\sigma_{\theta}^{2}$.

Second, differentiation with respect to $\sigma_{\varepsilon}^{2}$ yields

$$
\frac{\partial \Pi_{R}}{\partial \sigma_{\varepsilon}^{2}}=-\sqrt{\frac{1}{2 \pi}} \frac{\sigma_{\eta}^{2}-\tau}{\left(\sigma_{\varepsilon}^{2}+\sigma_{\eta}^{2}-\tau\right)^{3 / 2}}
$$

and

$$
\frac{\partial \Pi_{S}}{\partial \sigma_{\varepsilon}^{2}}=-\sqrt{\frac{1}{2 \pi}} \frac{\sigma_{\eta}^{2}-\tau}{\left(\sigma_{\varepsilon}^{2}+2 \sigma_{\eta}^{2}+2 \sigma_{\theta}^{2}\right)^{3 / 2}} .
$$

Comparison of these two results shows that $\Delta$ is decreasing in $\sigma_{\varepsilon}^{2}$. Third, differentiation with respect to $\sigma_{\eta}^{2}$ yields

$$
\frac{\partial \Pi_{R}}{\partial \sigma_{\eta}^{2}}=\sqrt{\frac{2}{\pi}} \frac{\sigma_{\varepsilon}^{2}+\frac{1}{2}\left(\sigma_{\eta}^{2}-\tau\right)}{\left(\sigma_{\varepsilon}^{2}+\sigma_{\eta}^{2}-\tau\right)^{3 / 2}}
$$

and

$$
\frac{\partial \Pi_{S}}{\partial \sigma_{\eta}^{2}}=\sqrt{\frac{2}{\pi}} \frac{\sigma_{\varepsilon}^{2}+\sigma_{\eta}^{2}+2 \sigma_{\theta}^{2}+\tau}{\left(\sigma_{\varepsilon}^{2}+2 \sigma_{\eta}^{2}+2 \sigma_{\theta}^{2}\right)^{3 / 2}} .
$$

Hence,

$$
\begin{aligned}
\frac{\partial \Pi_{R}}{\partial \sigma_{\eta}^{2}}>\frac{\partial \Pi_{S}}{\partial \sigma_{\eta}^{2}} \Leftrightarrow & \frac{2+(1-\rho) \alpha}{1+2 \beta+(1+\rho) \alpha}\left(\frac{1+2 \alpha+2 \beta}{1+(1-\rho) \alpha}\right)^{3 / 2}>2 \\
\Leftrightarrow & {[2+(1-\rho) \alpha]^{2}(1+2 \alpha+2 \beta)^{3} } \\
& -4[1+2 \beta+(1+\rho) \alpha]^{2}[1+(1-\rho) \alpha]^{3}>0,
\end{aligned}
$$

where $\alpha=\sigma_{\eta}^{2} / \sigma_{\varepsilon}^{2}, \beta=\sigma_{\theta}^{2} / \sigma_{\varepsilon}^{2}$, and $\rho=\tau / \sigma_{\eta}^{2}$.

This inequality can be rewritten as follows:

$$
\begin{gathered}
8 \beta(1+2 \beta)^{2}+\alpha^{2}\left(33+8 \beta^{3}(1-\rho)^{2}+12 \beta^{2}(1-\rho)(5+3 \rho)\right. \\
-\rho(10-9 \rho)+6 \beta[17-\rho(2-\rho)]) \\
+4 \alpha^{5}(1-\rho)^{2}\left(1-\rho+\rho^{2}+\rho^{3}\right) \\
+2 \alpha^{3}\left(23+4 \beta^{2}(1-\rho)^{2}(1+2 \rho)-\rho[18-(15-4 \rho) \rho]\right. \\
\left.\quad+4 \beta(1-\rho)\left(7+\rho+4 \rho^{2}\right)\right) \\
+4 \alpha^{4}(1-\rho)\left(6+2 \beta(1-\rho)\left(1+2 \rho^{2}\right)-\rho[4-\rho(5+\rho)]\right) \\
+8 \alpha(1+\beta[7+\rho+4 \beta(3+\beta-\beta \rho)])>0 .
\end{gathered}
$$

We have decomposed the right-hand side of the inequality into six terms, all of which can be shown to be positive using the fact that $0<\rho<1$. 


\section{ORTEGA}

Finally, differentiation with respect to $\tau$ shows that

$$
\frac{\partial \Pi_{R}}{\partial \tau}=-\sqrt{\frac{2}{\pi}} \frac{\sigma_{\varepsilon}^{2}+\frac{1}{2}\left(\sigma_{\eta}^{2}-\tau\right)}{\left(\sigma_{\varepsilon}^{2}+\sigma_{\eta}^{2}-\tau\right)^{3 / 2}}
$$

and

$$
\frac{\partial \Pi_{S}}{\partial \tau}=-\sqrt{\frac{2}{\pi}} \frac{1}{\left(\sigma_{\varepsilon}^{2}+2 \sigma_{\eta}^{2}+2 \sigma_{\theta}^{2}\right)^{1 / 2}} .
$$

Rearranging terms and using the definitions for $\alpha, \beta$, and $\rho$, we find

$$
\frac{\partial \Pi_{R}}{\partial \tau}<\frac{\partial \Pi_{S}}{\partial \tau} \Leftrightarrow \frac{1}{4}\left(\frac{2+(1-\rho) \alpha}{1+(1-\rho) \alpha}\right)^{2}>\frac{1+(1-\rho) \alpha}{1+2 \alpha+2 \beta} .
$$

The left-hand side of the inequality is monotonically increasing in $\rho$ and the right-hand side is monotonically decreasing in $\rho$. At $\rho=$ 1 the left-hand side is greater than the right-hand side, and $\Delta$ is decreasing in $\rho$. On the other hand, at $\rho=0$ the left-hand side might be greater or smaller than the right-hand side, depending on the values of the parameters.

Hence $\Delta$ might be monotonically decreasing in $\tau$ or might have an inverted $\mathrm{U}$ shape with respect to $\tau$.

\section{References}

Afonja, B. 1972. The moments of the maximum of correlated normal and $t$-variates. J. Royal Statist. Soc. B 34(2) 251-262.

Campion, M., L. Cheraskin, M. Stevens. 1994. Career-related antecedents and the outcomes of job rotation. Acad. Management J. 37(6) 1518-1542.
Gittleman, M., M. Horrigan, M. Joyce. 1998. "Flexible" workplace practices: Evidence from a nationally representative survey. Indust. Labor Relations Rev. 52(1) 99-115.

Ichniowski, C., K. Shaw. 1999. The effects of human resource management systems on economic performance: An international comparison of U.S. and Japanese plants. Management Sci. 45(5) 704-721.

G. Prennushi. 1997. The effect of human resource management practices on productivity. Amer. Econom. Rev. 87(3) 291-313.

, T. Kochan, D. Levine, C. Olson, G. Strauss. 1996. What works at work: Overview and assessment. Indust. Relations 35(3) 299-333.

Jovanovic, B. 1979. Job matching and the theory of turnover. J. Political Econom. 87(6) 972-990.

Meyer, M. 1994. The dynamics of learning with team production: Implications for task assignment. Quart. J. Econom. 109(4) 1157-1184.

Milgrom, P. 1988. Employment contracts, influence activities, and efficient organization design. J. Political Econom. 96(1) 42-60.

Miller, R. 1984. Job matching and occupational choice. J. Political Econom. 92(6) 1086-1120.

OECD. 1999. OECD Employment Outlook. Organisation for Economic Cooperation and Development, Paris, France.

Osterman, P. 1994. How common is workplace transformation and who adopts it? Indust. Labor Relations Rev. 47(2) 173-188.

2000. Work reorganization in an era of restructuring: Trends in diffusion and effects on employee welfare. Indust. Labor Relations Rev. 53(2) 179-196.

Pratt, J., H. Raiffa, R. Schlaifer. 1995. Statistical Decision Theory. The MIT Press, Cambridge, MA.

Accepted by Linda Argote; received November 14, 1999. This paper was with the author 7 months for 2 revisions. 